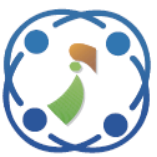

\title{
Optimal Network Reconfiguration and DG Integration in Power Distribution Systems Using Enhanced Water Cycle Algorithm
}

\author{
Sarmad Ibrahim ${ }^{1 *}$ \\ Shamam Alwash ${ }^{1}$ \\ Ahmed Aldhahab ${ }^{1}$ \\ ${ }^{1}$ Department of Electrical Engineering, University of Babylon, Babylon, Iraq \\ * Corresponding author's Email: sarmad_ibrahim1975@uky.edu
}

\begin{abstract}
This paper presents an Enhanced Water Cycle Algorithm (EWCA) to optimize the network reconfiguration and distributed generation (DG) integration simultaneously for minimizing system power losses and improving voltage stability index (VSI) in the distribution system while considering all operational constraints. For validation, the performance of the proposed method is compared with other methods, which utilized well-known meta-heuristic algorithms. Different cases for network reconfiguration and DG integration are carried out in order to evaluate the performance of the proposed method. The effectiveness of the proposed method is assessed using the IEEE 69-node radial distribution system. According to the simulation results obtained, the proposed method in which the simultaneous optimal network reconfiguration and the DG size and location are implemented can provide a remarkable solution in terms of power loss reduction and voltage profile improvement. The proposed method also proved its superiority compared with other existing methods in terms of power loss reduction (i.e., 84.2\% loss reduction compared with the base case).
\end{abstract}

Keywords: Enhanced water cycle algorithm, Optimal distribution network reconfiguration, Optimal DG integration, Power loss reduction, Voltage stability index.

\section{Introduction}

The increasing complexity of radial distribution systems due to the fast growth of the loads has caused significant challenges to the distribution utilities, such as poor voltage regulation and high-power system losses [1]. To address these challenges, a network reconfiguration strategy has become a viable technical solution for distribution system operators to perform fast voltage regulation and improve the overall performance of distribution systems [2]. In general, the distribution systems are equipped with sectionalizing and tie switches to alter the network topology of the distribution systems. These switches are designed to be either normally closed (i.e., sectionalizing switch) or opened (i.e., tie switches). The status of these switches can be changed manually or remotely to obtain the optimal network configuration that satisfies operational constraints with a significant improvement in overall voltage profile and reduction in system losses [3]. In the past decades, many researchers have preferred using only the network reconfiguration to improve the performance of distribution systems. These researchers have believed that other methods such as capacitor placement and cable size upgrading might add more additional cost burdens to the distribution system utilities. In [4-7], the system losses and voltage profile are improved by formulating the network reconfiguration problem as a mixed-integer nonlinear optimization problem using various metaheuristic algorithms with considering operational constraints. Due to the rapid growth of the integration of the distributed generations (DGs) into the distribution system, the use of the network reconfiguration technique without considering the presence of the DGs is no longer applicable. The optimal DG installation in the distribution can effectively contribute to improving the performance of the distribution systems in terms of system loss reduction and voltage profile enhancement [8]. 
Several approaches have been proposed to reduce system losses and enhance the overall voltage profile in the distribution system in the presence of the DG.

C. K. Das and O. Bass [9] developed a new strategy for optimal placement of distributed energy storage systems based on the artificial bee colony approach to mitigate network losses and line loading. D.B. Prakash and C. Lakshminarayana [10] proposed optimal placement and sizing of DGs in the distribution system using an analytical method and whale optimization algorithm. P. Mehta, P. Bhatt, and V. Pandya [11] presented a new strategy for the optimal DG size and location based on a comprehensive analytical method to improve the voltage stability of the distribution system. Although the approaches mentioned above have relatively enhanced the performance of distribution systems, such approaches have only focused on the integration of the DG location and size without considering the network reconfiguration in distribution systems.

With the aim to obtain a further improvement in the distribution system performance, several researchers have adopted that the simultaneous optimal network reconfiguration and DG size and location can bring more benefits for distribution utilities in terms of the system loss reduction and voltage profile [12-18]. For example, M. S. Rawat and S. Vadhera employed an approach to optimize the system performance in terms of system loss reduction and voltage profile improvement. In this approach, Particle Swarm Optimization (PSO) is used for solving the optimal reconfiguration with the concurrent DG installation [12]. Z. Gong, Q. Chen, and K. Sun [13] aimed to reduce the system loss and improve the voltage stability of the radial distribution system using an Electromagnetism-like Mechanism (ELM) optimization method for solving the distribution system reconfiguration problem with the DG size and location. R. S. Rao, K. Ravindra, K. Satish, and S. V. L. Narasimham [14] proposed a method to find the best distribution system configuration by taking into account the optimal DG installation based on Harmony Search Algorithm (HSA). A. M. Imran, M. Kowsalya, and D.P. Kothari [15] used Fireworks Algorithm (FWA) for optimally solving distribution system reconfiguration and the DG placement simultaneously. The main objective of this study is to minimize the system loss and improve the voltage stability of the distribution system. T. T. Nguyen, A. V. Truong, and T. A. Phung [16] applied Adaptive Cuckoo Search Algorithm (ACSA) and graph theory to find the optimal distribution system configuration and the DG size and location with considering the system loss and voltage stability index as an objective function. A Strength Pareto
Evolutionary Algorithm was proposed by I. B. Hamida, S. B. Salah, F. Msahli, and M. F. Mimouni [17] to reduce system losses and annual operation costs, considering simultaneous optimal network reconfiguration and DG size and location. Despite the simulation results obtained from these approaches illustrated the encouraging improvement of the distribution system performance, searching for better performance of the distribution system is requisite. Accordingly, the enhanced form of Water Cycle Algorithm (WCA) is proposed herein to provide further improvement of the distribution system performance depending on a remarkable searching process for a global solution.

Recently, a Water Cycle Algorithm (WCA) has been successfully applied in different fields to solve complex linear and non-linear optimization problems with high accuracy and fast convergence speed [19]. Several researchers have used and modified the WCA to tackling the optimization problems with either a continuous or discrete search space [19-23]. For instance, K. Yanjun, M. Yadong, L. Weinan, W. Xianxun, and B. Yue [23] used the chaos-based method and a dynamic adaptive parameter to enhance the WCA performance in terms of an initial population and exploration for better search performance. However, this modification only considered continuous variables.

The network reconfiguration problem with the DG location and size considered herein is a complex combinatorial optimization problem when its variables are discrete and continuous.

In this paper, the search performance of the original WCA is enhanced to deal with both discrete and continuous search spaces simultaneously. The Enhanced Water Cycle Algorithm (EWCA) aims to attain the best optimum solution for distribution system reconfiguration and the DG size and location that satisfy significant power loss reduction and voltage stability improvement while maintaining all operational constraints with acceptable limits. In addition, different cases and comparisons are presented to verify and highlight the effectiveness of the proposed method in terms of system loss reduction and voltage stability improvement, with and without considering the presence of DGs in the distribution system.

The remainder of this paper is organized as follows. Section 2 provides a description of the problem formulation. Section 3 illustrates the main steps of the EWCA. Section 4 describes the test system. In Section 5, the simulation results and discussion are provided. Finally, the conclusion is outlined in Section 6. 


\section{Problem formulation}

The proposed method based on EWCA seeks to minimize the system loss and improve the voltage stability of the distribution system. In this study, the power loss improvement index and the voltage stability index are combined to construct the proposed objective function with considering simultaneous optimal network reconfiguration and DG integration. In general, the total active power loss $P_{\text {loss }_{T}}$ dissipating in the radial distribution system is equal to the summation of the loss in all branches, which can be defined as follows:

$$
P_{\text {loss }_{T}}=\sum_{d \in N_{B}} q_{d} P_{\text {loss }}(d)
$$

where $N_{B}$ is the total number of branches, $q_{d}$ represents the status of the branch $d$ (i.e., either branch $d$ is unconnected $q_{d}=0$ or connected $q_{d}=1$ ), and $P_{\text {loss }}(d)$ is the active power loss in the branch $d$. The power loss improvement index $\Delta P_{\text {loss }}^{\text {index }}$ is calculated by the ratio of total active power loss before and after the system improvement [24], which is given by

$$
\Delta P_{\text {loss }}^{\text {index }}=\frac{P_{\text {loss }_{T}}^{\mu}}{P_{\text {loss }_{T}}^{v}}
$$

where $P_{\text {loss }}^{\mu}$ and $P_{\text {loss }_{T}}^{v}$ are the total active power loss of the distribution system after and before the system improvement, respectively.

The performance of the distribution systems can be evaluated using the Voltage Stability Index (VSI). The VSI is mainly used to study the distribution system behavior in terms of the voltage stability margin and discover the possible instability of operating points of system nodes. The critical voltage stability of the distribution system can cause poor voltage regulation and high system losses. Therefore, it is essential to measure the VSI of the distribution system and find the nearest possible point to have voltage collapse. According to [25, 26], the VSI formula at node $j$ can be represented as follows:

$$
\begin{aligned}
\operatorname{VSI}(\mathrm{j})=\left|V_{i}\right|^{4}- & 4\left(P_{j} X_{i j}-Q_{j} R_{i j}\right)^{2} \\
& -4\left(P_{j} R_{i j}-Q_{j} X_{i j}\right)\left|V_{i}\right|^{2}
\end{aligned}
$$

where $V_{i}$ is a voltage at node $i,(i, j) \in N_{\text {node }}, N_{\text {node }}$ represents the total number of nodes, $P_{j}$ and $Q_{j}$ represent active and reactive power load drawn from node $j$, respectively, and $R_{i j}$ and $X_{i j}$ are resistance and reactance of the branch connected between node $i$ and $j$, respectively. The minimum VSI can be calculated using Eq. (4)

$$
\mathrm{VSI}_{\text {min }}=\min (\mathbf{V S I})
$$

In this paper, the power loss improvement index and the voltage stability index are combined in order to form the objective function, which is defined as:

$$
\min _{\mathbf{H}}\left(w_{1} \Delta P_{\text {loss }}^{\text {index }}+w_{2}\left(1-\operatorname{VSI}_{\min }\right)\right)
$$

where $\mathbf{H}=[\mathbf{X}, \mathbf{L}, \mathbf{Z}]$ is a vector that represents an initial candidate solution, $\mathbf{X}, \mathbf{L}$, and $\mathbf{Z}$ are row vectors containing the status of the branch and tie switches, the size of DGs, and the DG locations, respectively, and $w_{1}$ and $w_{2}$ are weighted coefficients used to convert a multi-objective optimization problem to a single optimization problem. The objective function indicated in Eq. (5) relatively focuses on a loss reduction (i.e., $w_{1}=0.7$ ) and voltage stability (i.e., $w_{2}=0.3$ ) of the distribution system. The status of the sectionalizing and tie switch vector $\mathbf{X}$, the DG location vector $\mathbf{L}$, and the size of the DGs $\mathbf{Z}$ can be expressed as:

$$
\begin{gathered}
\mathbf{X}=\left\{b_{1}, b_{2}, b_{n}, \ldots, t_{1}, t_{2}, \ldots, t_{m}\right\} \\
\mathbf{L}=\left\{l_{1}, l_{2}, \ldots, l_{n g}\right\} \\
\mathbf{Z}=\left\{z_{1}, z_{2}, \ldots, z_{n g}\right\}
\end{gathered}
$$

where $b$ and $t$ represents the sectionalizing and tie switches, respectively, $n$ and $m$ are the total numbers of sectionalizing and tie switches equipped with the distribution system, respectively, $l$ denotes the node number where the DG should be placed, and $l \in$ $\left\{1,2, \ldots, N_{\text {node }}\right\}, n_{g}$ is the total number of DGs integrated into a distribution system, and $z$ refers to the size of the DG injected at the specified node.

The specified equality and inequality constraints of the objective function that should be emphasized are classified as follows: for inequality constraints, the considered node voltage magnitudes of the distribution system must be kept within acceptable limits:

$$
\mathbf{V}_{l r} \leq \widetilde{\mathbf{V}} \leq \mathbf{V}_{u r}
$$

where $\mathbf{V}_{l r}$ and $\mathbf{V}_{u r}$ represent the lower and upper limits of the voltage magnitude, respectively. The 
voltage magnitude limits are selected to be $0.95 \mathrm{p} . \mathrm{u}$ and 1.05 p.u based on ANSI standard in the U.S. [27]. In this paper, the branch current limits are also considered as an inequality constraint, which can be defined as:

$$
\left|I_{d}\right| \leq I^{\max }
$$

where $I_{d}$ represents the branch current passing through branch $d$ and $I^{\max }$ is the maximum current of each branch. For equality constraints, the active power balance constraint must be satisfied, which can be defined as

$$
P_{\text {loss }_{T}}+P_{\text {load }_{T}}=P_{\text {sub }}+\sum_{k \in n g} P_{G_{k}}
$$

where $P_{\text {load }}$ is the total load connected into the distribution system, $P_{G_{k}}$ is the active power injected by the DG at node $k$, and $P_{\text {sub }}$ represents the total active power drawn from the main substation. A radiality of the distribution system must be kept over the network reconfiguration to guarantee all loads are remained connected and energized from the main substation. Therefore, the radiality constraint is included in the proposed objective function as an equality constraint, which can be expressed as follows:

$$
q_{d} \times N_{B}=N_{\text {node }}-1
$$

\section{Description of the proposed EWCA}

Network reconfiguration in distribution systems considering the DG location and size is a complex nonlinear optimization problem. This type of optimization problem cannot be solved using conventional techniques. Therefore, the WCA is enhanced for solving a network reconfiguration problem taking into account the DG location and size simultaneously into the distribution system. In this paper, the main advantage of the EWCA proposed is its ability to attain a global solution with the desired convergence speed and accuracy. Moreover, the proposed algorithm can efficiently solve the system reconfiguration problem with considering the DG location and size and considering the discrete and continuous nature of the controlled variables.

In general, the Water Cycle Algorithm was firstly proposed by [19]. According to [19], the metaheuristic method is established based on observing the movement of water in nature. The water cycle process begins its natural circulation from raindrops that are formed from the evaporation process. Then raindrops have gradually clustered to form streams, several of which in turn form rivers. In nature, streams and rivers move towards the sea. However, some streams might flow either to form rivers or directly towards the sea. The main steps of the EWCA are summarized as follows:

\subsection{Generation of an initial population}

Like any population-based meta-heuristic algorithm, the first step to solve any optimization problem is to generate a random initial population matrix. As mentioned before, the raindrops are the first stage of the water cycle in nature to produce streams, rivers, and sea. In the EWCA, an initial population matrix $\mathbf{D}$ is created by raindrops. Each raindrop or row in the matrix $\mathbf{D}$ is considered as an initial candidate solution for the proposed optimization problem. In this mechanism, an initial candidate solution represents the controlled variables of the optimization problem. To satisfy the constraints of the optimization problem, the controlled variables are randomly initiated between upper and lower boundaries. This initial population matrix $\mathbf{D}$ can be defined as:

$$
\mathbf{D}=\left[\begin{array}{c}
D_{1} \\
D_{2} \\
\vdots \\
D_{N_{p}}
\end{array}\right]
$$

where $D_{x}=\left[r_{x 1}, r_{x 2}, \ldots, r_{x z}\right]$ represents the raindrop, $x \in N_{p}, N_{p}$ is the population size, $z \in N_{v}$, and $N_{v}$ is the total number of the controlled variables. Each raindrop in the initial population matrix $\mathbf{D}$ is encoded as shown in Fig.1. Fig.1 illustrates the binary controlled variables represented by the status of sectionalizing and tie switches, the integer-controlled variables denoted by the DG locations, and the real controlled variables indicated by the DG sizes.

\subsection{Evaluation and classification of raindrops}

The cost value of each raindrop in matrix $\mathbf{D}$ is evaluated using the cost function indicated in Eq. (14)

$$
\operatorname{Cost}_{x}=f\left(D_{x}\right)
$$

Subsequently, these evaluated raindrops are

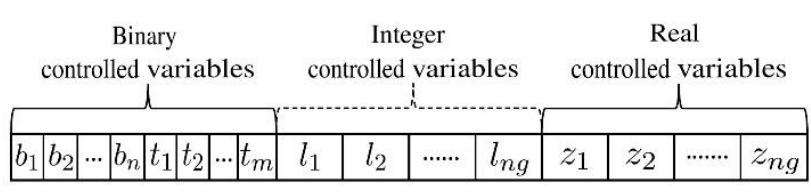

Figure. 1 The encoded raindrop used by the EWCA 
classified in an ascending order based on their cost values. The raindrop that has the minimum value is selected as the sea. The next best cost values are chosen as river while the rest of the cost values are selected as streams.

$$
\begin{gathered}
N_{s a}=S e a+N_{r i} \\
N_{p}=N_{s a}+N_{s r}
\end{gathered}
$$

where $N_{r i}$ and $N_{s r}$ represent the total number of rivers and streams, respectively.

\subsection{Calculation the flow intensity of streams}

Each river and sea naturally absorb their water from streams. Therefore, it is essential to determine the flow intensity of the designed streams that either flow to rivers or immediately flow to sea [19], which can be expressed as:

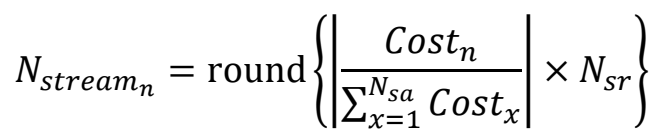

where $N_{\text {stream }}$ is the number of designed streams, which flow to the specific rivers and sea and $n \in N_{s a}$.

\subsection{New positions of streams and rivers}

The positions of streams and rivers are changed continuously to diverse the population and avoid getting trapped in a local optimum. According to [19], the positions of streams and rivers are updated based on a continuous search space to solve the optimization problems that only have real controlled variables. However, the proposed problem herein is a non-linear combinatorial optimization problem, having simultaneous continuous and discrete controlled variables. In order to address this problem, several modified WCAs have been proposed to solve a non-linear optimization problem with either continuous or discrete control variables [19-23]. However, none of these algorithms handled both discrete and continuous controlled variables for the same optimization problem. In this paper, the uniform crossover operation technique is proposed to deal with both discrete and continuous controlled variables. The uniform crossover operation technique is separately applied for each part of the designed raindrop shown in Fig.1 (i.e., the binary, integer, and real controlled variables).

\section{Evaporation and raining process}

During the search for the global optimum solution, the distance between the sea and rivers becomes very small. In other words, the search ends up with no further improvement in the solution quality due to being trapped in a local optimum solution. Therefore, the evaporation process is an essential condition of the EWCA to prevent this algorithm from a fast convergence and being trapped in local optima. In this stage, the evaporation condition is used to determine whether streams or rivers arrived or sufficiently close to the sea. For this purpose, Eq. (18) is used to check the evaporation condition between the sea and rivers [22]:

$$
\left\|\mathbf{X}_{\text {sea }}^{\beta}-\mathbf{X}_{\text {river }}^{\beta}\right\|<d_{\max }
$$

where $d_{\max }$ is a predefined value near to zero, $\beta \in$ $\left\{1,2, \ldots\right.$, Max $\left._{i t e r}\right\}$, and Max $_{i t e r}$ is the total number of iterations. The value of $d_{\max }$ is utilized to control the search intensity near the sea to obtain an optimal solution. This predefined value is iteratively updated during iterations, which can be defined as:

$$
d_{\text {max }}^{\beta+1}=d_{\text {max }}^{\beta}-\frac{d_{\text {max }}^{\beta}}{\text { Max }_{\text {iter }}}
$$

Once the evaporation condition is satisfied, the raining process immediately starts to form new raindrops, which lead to generating new streams in different locations. In this paper, a mutation operator is used to perform the raining process based on a random probability, which can be expressed as:

$$
\mathbf{X}_{\text {stream }}^{\text {new }}=\mathbf{X}_{l b}+\text { rand } \times\left(\mathbf{X}_{u b}-\mathbf{X}_{l b}\right)
$$

where $\mathbf{X}_{l b}$ and $\mathbf{X}_{u b}$ represent the vectors containing upper and lower limits of the control variables, and rand is a random number between 0 and 1 .

The main procedure of the EWCA shown in Fig. 2 is utilized to minimize the system loss and improve voltage stability through the simultaneous optimal network reconfiguration and the DG installation.

\section{Test system description}

The performance of the proposed method is tested on the IEEE 69-node radial distribution system [28] shown in Fig. 3. This distribution system consists of 69 nodes and 73 branches, has normally opened tie switches represented by $T_{69}, T_{70}, T_{71}, T_{72}$ and $T_{73}$ and indicated by dotted lines, and has normally closed sectionalizing switches numbered from $B_{1}$ to $B_{68}$ and indicated by solid lines. The nominal voltage 


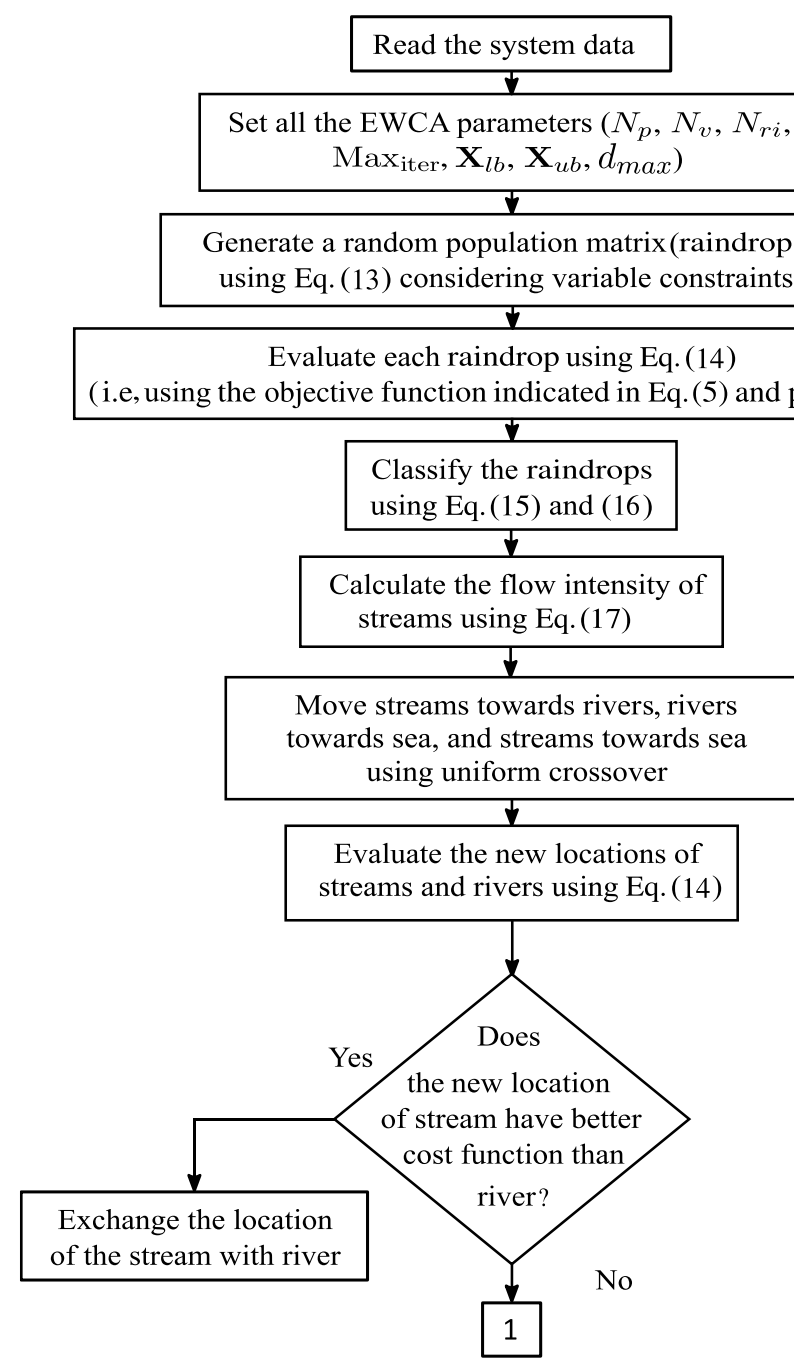

Figure. 2 The flow chart for the EWCA

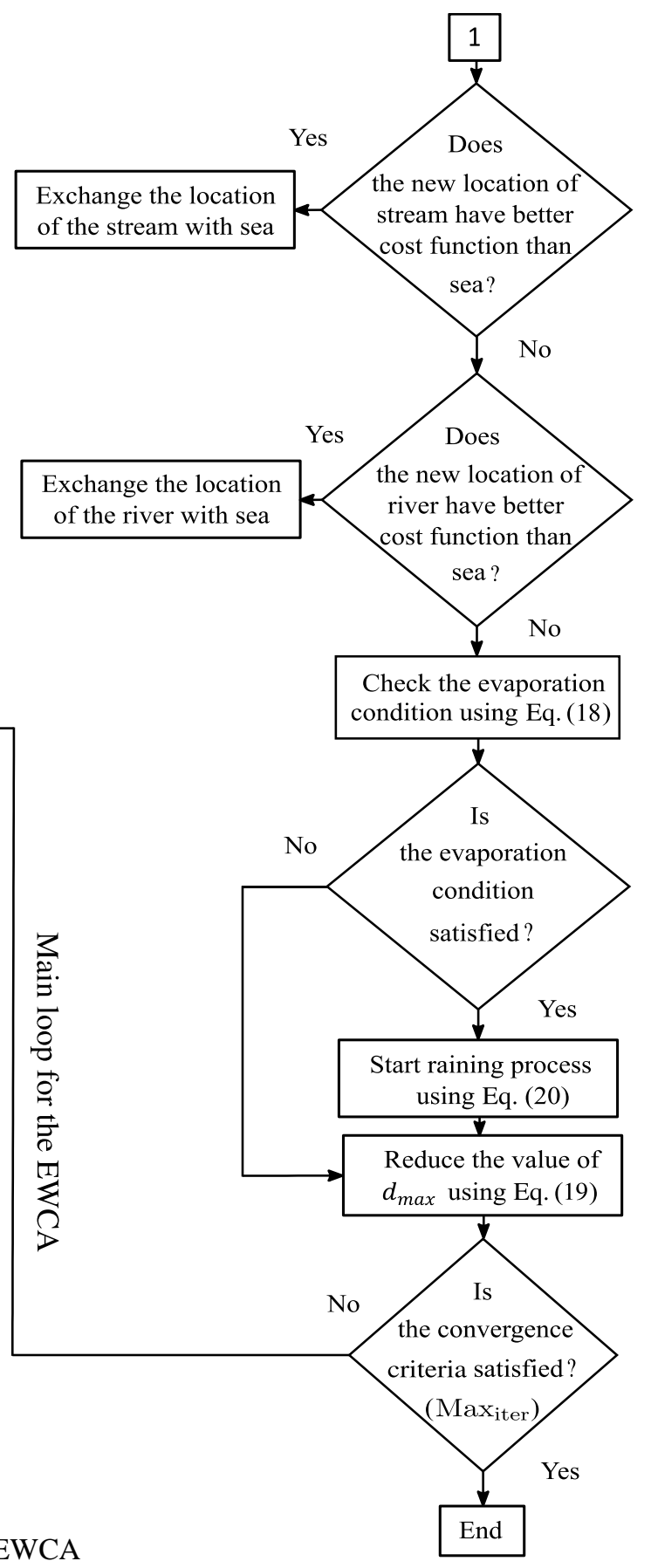

used is set to be $12.66 \mathrm{kV}$ (or 1 p.u.), and the total system load is $3.8 \mathrm{MW}$ and 2.70 MVAr.

To examine the effectiveness of the proposed method, four different cases are carried out to minimize the system loss and improve the voltage profile based on the EWCA, while considering all system constraints. These cases, including the base case, are categorized as follows:

Base case: The distribution system is without considering the network reconfiguration and the DG installation.

Case 1: It is similar to conventional methods in which network reconfiguration is only considered [4].
Case 2: This case is similar to approaches in which the optimal DG size and location are optimized without performing the network reconfiguration [11]. Case 3: The optimal network reconfiguration simultaneously with only the DG size is performed [14]. In this case, the VSI is firstly utilized to determine the weakest nodes in terms of the voltage stability index. These nodes are chosen to be the best candidate location for the DG active power injection. Case 4: This case represents the proposed method in which the simultaneous optimal network reconfiguration and the DG size and location are performed to improve the performance of the 


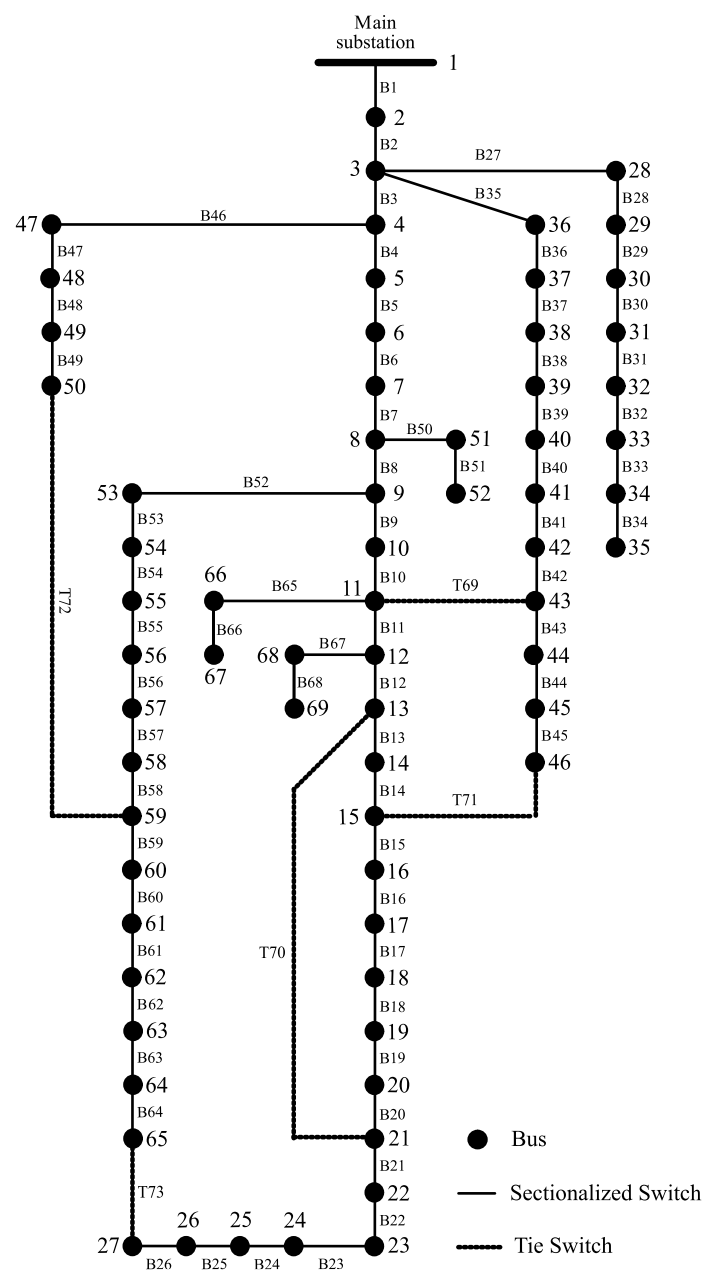

Figure. 3 IEEE 69-node radial distribution system

distribution system in terms of loss reduction and voltage profile improvement.

In this paper, the superiority of the proposed method in terms of the loss reduction is verified by comparing it with other existing methods, which used well-known meta-heuristic algorithms and adopted the same proposed problem mentioned in case 4 . These existing methods used the Particle Swarm Optimization (PSO) [12], the Electromagnetism Like mechanism (ELM) [13], the Harmony Search Algorithm (HSA) [14], the Fireworks Algorithm (FWA) [15], and the Adaptive Cuckoo Search Algorithm (ACSA) [16]. In this paper, a power flow technique [29] is used for evaluating the feasibility of candidate solutions and determining the VSI, node voltages, branch currents, and system losses. There are three of the DG locations suggested to be installed in the distribution system. The limits of the DG sizes are chosen to be between 0 and $2 \mathrm{MW}$.

\section{Simulation results and discussions}

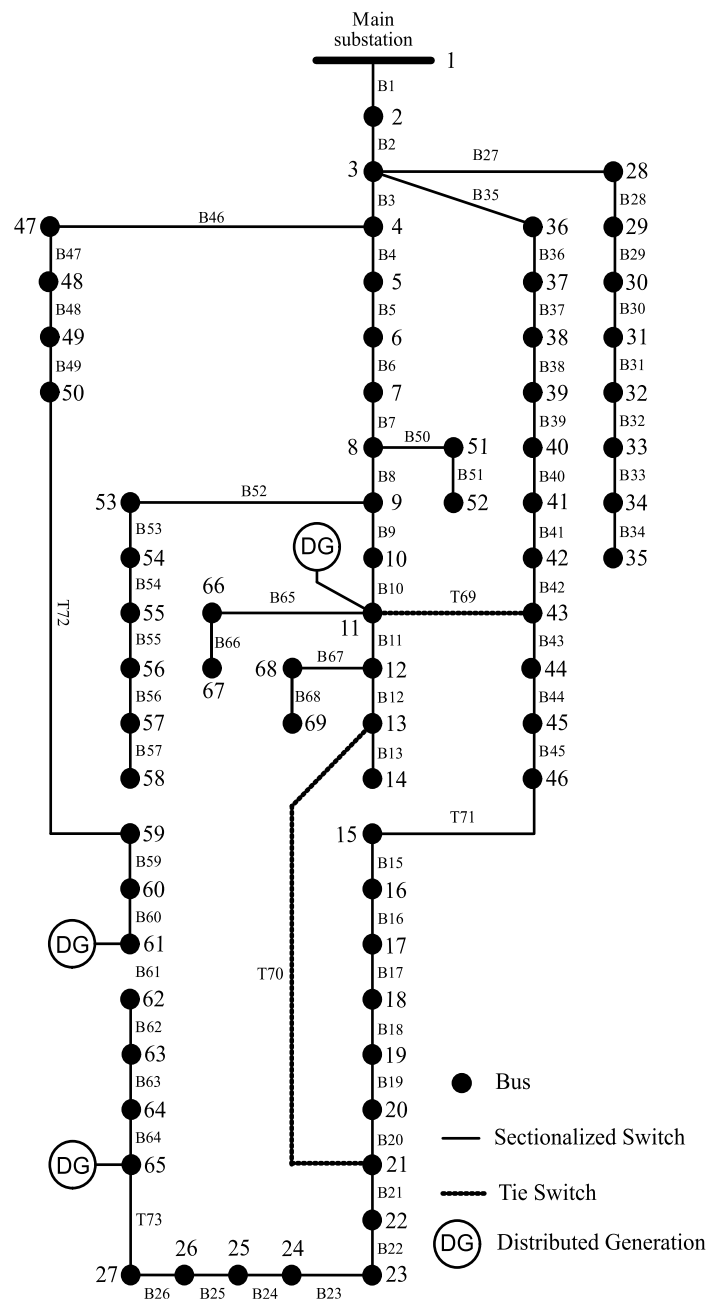

Figure. 4 The optimal network configuration and DG location for IEEE 69-node radial distribution system

The four cases mentioned above are simulated and presented in Table 1. As seen in Table 1, the base case is the case without any improvement in the performance of the test system. This case is the worst case in terms of the loss reduction, the minimum VSI, and the voltage magnitude violation. The case 1 is also presented in Table 1. In this case, the optimal reconfiguration selected by the EWCA is to open the sectionalizing switches $14,57,61$, and to close the tie switches 71, 72, 73. From the simulation results obtained for the case 1 , it is observed that the system loss is significantly improved by $55.73 \%$ while the minimum voltage magnitude and the minimum VSI have a modest improvement, which is nearly $3.7 \%$ and $15.75 \%$, respectively, compared with the base case. This is expected because this case focuses on the optimal network reconfiguration without considering the voltage stability index.

Table 1 also shows that the use of the DGs with the penetration level $68 \%$ successfully improves the overall performance of the test system in case 2 compared with case 1 and base case. In this case, the 


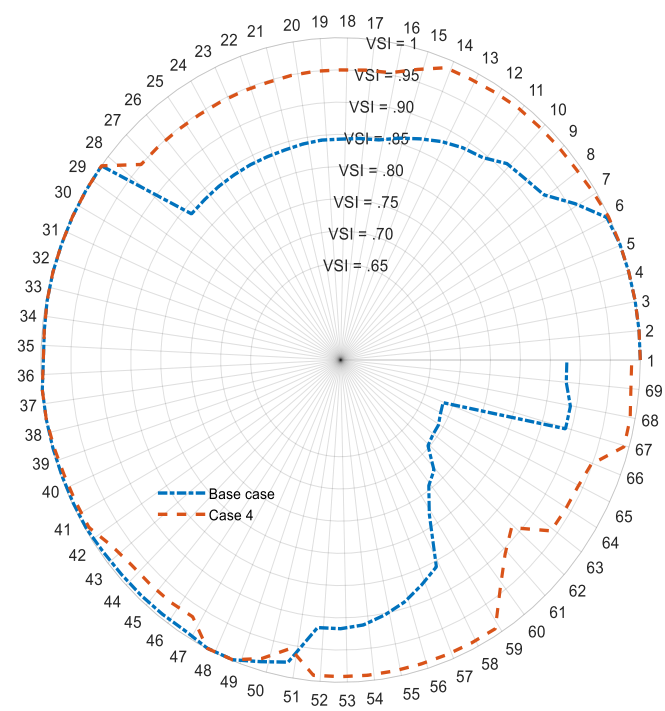

Figure. 5 Comparison of VSI for each node of 69-node test distribution system for case 4 and base case

system loss, the minimum voltage magnitude, and the minimum VSI are greatly enhanced by $69.1 \%, 7.5 \%$, and $33 \%$, respectively, compared with the base case. It can also be seen in Table 1 that the use of the VSI in case 3 to select the suitable DG locations can effectively participate in more loss reduction, which is $78.7 \%$ compared with the base case. According to results obtained for case 3 , the optimal reconfiguration of the test system is performed by opening the sectionalizing switches $9,12,13,55,64$, and closing the tie switches $69,70,71,72,73$. Also, for this case, the optimal penetration level chosen is $51.5 \%$. In addition, the minimum voltage magnitude and the minimum VSI are improved by $6.55 \%$ and $26.7 \%$, respectively, compared to the base case.

The proposed method represented by case 4 is also shown in Table 1. In this case, the optimal reconfiguration of the test system is presented in Fig. 4 in which the optimal sectionalizing switches are 14 , 58, 61 and the optimal tie switches are 71, 72, 73. The results in Table 1 show that the loss reduction and the improvement in the minimum voltage magnitude are $84.2 \%, 7.22 \%$, respectively, compared with the base case. Also, the VSI of the most system nodes is successfully improved as shown in Fig. 5 in which the minimum VSI is enhanced by $29.7 \%$, compared with the base case. The power loss reduction in case 4 is clearly illustrated through the decrease in the current magnitude of each branch of the distribution system as shown in Fig 6. Although the current magnitude in some branches increased due to the network reconfiguration as seen in Fig 6, the current magnitude is minimized in most of the branches of the test system.

The voltage profile curves for all cases, including the base case, are compared and presented in Fig. 7.

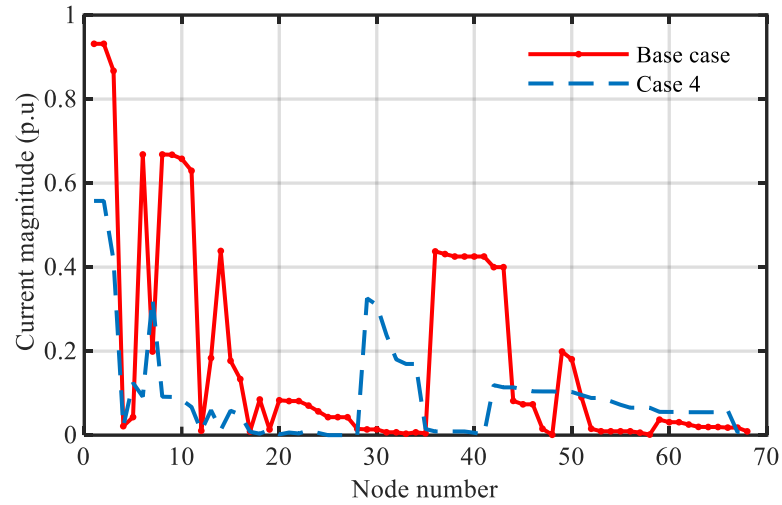

Figure. 6 Comparison of the current magnitude at each line of 69 -node test distribution system for case 4 and base case

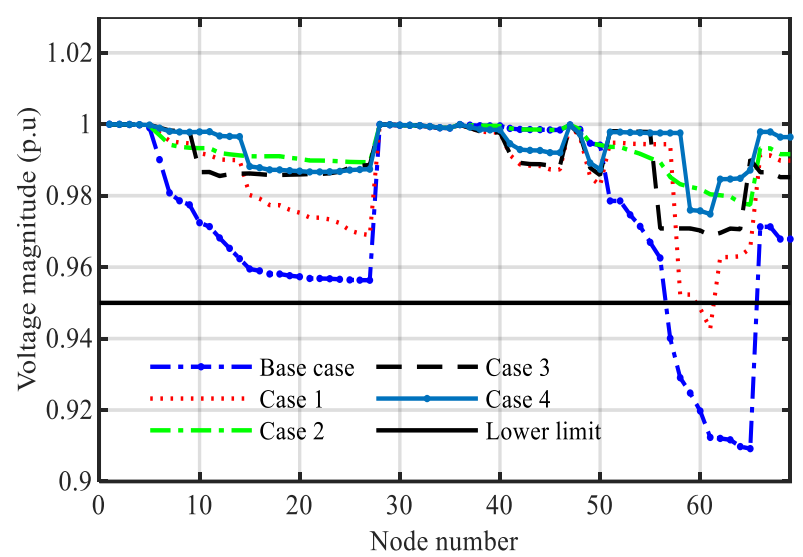

Figure. 7 Voltage magnitude profile of 69node test distribution system for all cases

This figure shows that the voltage magnitudes of all nodes in the test system are within acceptable limits, except the base case and case 1 . For case 1, this is expected because the search space is extremely restricted by a radial topology of the test system. Consequently, this case is not able to find the optimal solution with maintaining all system nodes within permissible ranges. Also, from this figure, it can be observed that the case 4 presents the best voltage profile improvement compared with the other cases. What can be concluded from the preceding results and discussions is that the proposed method represented in case 4 has outperformed the other existing methods adopted the same approaches.

Table 2 refers to the comparison between the proposed method described in the case 4 and the existing ones presented in the literature in terms of the optimal system configuration, the optimal DG size and location, and the power loss. Despite the proposed method and ACSA having the same network configuration and the DG placements, as shown in Table 2, the proposed method has optimally selected the DG sizes to be more effective in system loss reduction. It can also be seen from Table 2 that the proposed method has outperformed the other 
Table 1. Simulation results of the proposed EWCA on the 69-node test distribution system

\begin{tabular}{|c|c|c|c|c|c|}
\hline Cases & $\begin{array}{c}\text { Optimal } \\
\text { Switch } \\
\text { configuration }\end{array}$ & $\begin{array}{c}\text { Optimal } \\
\text { DG Size }(\mathrm{MW}) / \\
\text { location }\end{array}$ & $\begin{array}{c}\text { Power } \\
\text { Loss } \\
\text { (MW) }\end{array}$ & $\begin{array}{c}\text { Minimum } \\
\text { Voltage } \\
\text { (p.u) }\end{array}$ & $\begin{array}{l}\text { Minimum } \\
\text { VSI }\end{array}$ \\
\hline Base case & Opened switches $\left(\mathrm{T}_{69}, \mathrm{~T}_{70}, \mathrm{~T}_{71}, \mathrm{~T}_{72}, \mathrm{~T}_{73}\right)$ & - & 224.96 & 0.9092 & 0.6833 \\
\hline Case 1 & $\begin{array}{l}\text { Opened switches }\left(\mathrm{B}_{14}, \mathrm{~B}_{57}, \mathrm{~B}_{61}\right) \\
\text { Closed switches }\left(\mathrm{T}_{71}, \mathrm{~T}_{72}, \mathrm{~T}_{73}\right)\end{array}$ & - & 99.60 & 0.9428 & 0.7897 \\
\hline Case 2 & Opened switches $\left(\mathrm{T}_{69}, \mathrm{~T}_{70}, \mathrm{~T}_{71}, \mathrm{~T}_{72}, \mathrm{~T}_{73}\right)$ & $\begin{array}{l}0.5422 / 11 \\
0.3808 / 18 \\
1.6725 / 61\end{array}$ & 69.51 & 0.9775 & 0.9087 \\
\hline Case 3 & $\begin{array}{l}\text { Opened switches }\left(\mathrm{B}_{9}, \mathrm{~B}_{12}, \mathrm{~B}_{13}, \mathrm{~B}_{55}, \mathrm{~B}_{64}\right) \\
\text { Closed switches }\left(\mathrm{T}_{69}, \mathrm{~T}_{70}, \mathrm{~T}_{71}, \mathrm{~T}_{72}, \mathrm{~T}_{73}\right)\end{array}$ & $\begin{array}{l}1.3399 / 63 \\
0.2685 / 64 \\
0.3478 / 65\end{array}$ & 47.90 & 0.9688 & 0.8659 \\
\hline Case 4 & $\begin{array}{l}\text { Opened switches }\left(\mathrm{B}_{14}, \mathrm{~B}_{58}, \mathrm{~B}_{61}\right) \\
\text { Closed switches }\left(\mathrm{T}_{71}, \mathrm{~T}_{72}, \mathrm{~T}_{73}\right)\end{array}$ & $\begin{array}{l}0.5413 / 11 \\
1.4236 / 61 \\
0.5084 / 65\end{array}$ & 35.50 & 0.9749 & 0.8859 \\
\hline
\end{tabular}

Table 2. Simulation results of the proposed EWCA on the 69-node test distribution system for Case 4 in a comparison with existing methods

\begin{tabular}{|c|c|c|c|}
\hline Methods & $\begin{array}{c}\text { Optimal } \\
\text { Switch } \\
\text { configuration }\end{array}$ & $\begin{array}{c}\text { Optimal } \\
\text { DG Size }(\mathrm{MW}) / \\
\text { location }\end{array}$ & $\begin{array}{c}\text { Power } \\
\text { Loss } \\
\text { (MW) }\end{array}$ \\
\hline PSO & $\begin{array}{l}\text { Opened switches }\left(\mathrm{B}_{9}, \mathrm{~B}_{12}, \mathrm{~B}_{58}, \mathrm{~B}_{62}\right) \\
\text { Closed switches }\left(\mathrm{T}_{69}, \mathrm{~T}_{71}, \mathrm{~T}_{72}, \mathrm{~T}_{73}\right)\end{array}$ & $\begin{array}{l}0.8834 / 23 \\
1.0227 / 38 \\
1.3403 / 61 \\
\end{array}$ & 41.85 \\
\hline ELM & $\begin{array}{l}\text { Opened switches }\left(\mathrm{B}_{9}, \mathrm{~B}_{12}, \mathrm{~B}_{16}, \mathrm{~B}_{55}, \mathrm{~B}_{63}\right) \\
\text { Closed switches }\left(\mathrm{T}_{69}, \mathrm{~T}_{70}, \mathrm{~T}_{71}, \mathrm{~T}_{72}, \mathrm{~T}_{73}\right)\end{array}$ & $\begin{array}{l}0.7023 / 22 \\
1.4372 / 61 \\
0.6489 / 67 \\
\end{array}$ & 40.89 \\
\hline HSA & $\begin{array}{l}\text { Opened switches }\left(\mathrm{B}_{13}, \mathrm{~B}_{17}, \mathrm{~B}_{58}, \mathrm{~B}_{61}\right) \\
\text { Closed switches }\left(\mathrm{T}_{70}, \mathrm{~T}_{71}, \mathrm{~T}_{72}, \mathrm{~T}_{73}\right)\end{array}$ & $\begin{array}{l}0.3525 / 60 \\
1.0666 / 61 \\
0.4527 / 62\end{array}$ & 40.30 \\
\hline FWA & $\begin{array}{l}\text { Opened switches }\left(\mathrm{B}_{13}, \mathrm{~B}_{55}, \mathrm{~B}_{63}\right) \\
\text { Closed switches }\left(\mathrm{T}_{71}, \mathrm{~T}_{72}, \mathrm{~T}_{73}\right)\end{array}$ & $\begin{array}{l}1.1272 / 61 \\
0.2750 / 62 \\
0.4159 / 65\end{array}$ & 39.25 \\
\hline ACSA & $\begin{array}{l}\text { Opened switches }\left(\mathrm{B}_{14}, \mathrm{~B}_{58}, \mathrm{~B}_{61}\right) \\
\text { Closed switches }\left(\mathrm{T}_{71}, \mathrm{~T}_{72}, \mathrm{~T}_{73}\right)\end{array}$ & $\begin{array}{l}0.5413 / 11 \\
1.7240 / 61 \\
0.5536 / 65 \\
\end{array}$ & 37.02 \\
\hline EWCA & $\begin{array}{l}\text { Opened switches }\left(\mathrm{B}_{14}, \mathrm{~B}_{58}, \mathrm{~B}_{61}\right) \\
\text { Closed switches }\left(\mathrm{T}_{71}, \mathrm{~T}_{72}, \mathrm{~T}_{73}\right)\end{array}$ & $\begin{array}{l}0.5413 / 11 \\
1.4236 / 61 \\
0.5084 / 65\end{array}$ & 35.50 \\
\hline
\end{tabular}

methods in terms of the loss reduction compared to the base case.

To end this, Tables 1 and 2 prove that the proposed EWCA achieved a more effective solution in terms of loss reduction and the voltage profile improvement than other comparative cases when the network reconfiguration and the DG integration are simultaneously optimized. Another conclusion that can be observed from obtained simulation results is that the proposed EWCA has attained a better solution with high superiority in terms of loss reduction with acceptable voltage profile improvement compared with other existing methods. The proposed EWCA outperforms the other existing methods that adopted different metaheuristic algorithms because of choosing a conceptually different approach in finding the global optimum solution. Although the compared algorithms 
mentioned and the proposed EWCA primarily depend on population-based metaheuristic algorithms, the process in attaining the global solution differs from one algorithm to the other. The proposed EWCA uniquely utilize s the indirect path to explore the global optimum solution by updating the position of streams and rivers towards the best temporary optimum solution represented as the sea. Moreover, the proposed EWCA adopts the evaporation and raining process, which avoids the EWCA trapping in a local optimum solution [21]. This process is repeated until finding the global optimum solution.

\section{Conclusion}

This paper presents an Enhanced Water Cycle Algorithm (EWCA) for solving a complex network reconfiguration with considering the DG size and location. This algorithm uses a uniform crossover operating technique for simultaneously dealing with the discrete and continuous search space, compared with other algorithms that deal with either the discrete or continuous search space. The considered objective function of the proposed method has focused on the loss reduction and the voltage stability margin simultaneously in the distribution system while considering all operational constraints.

Different cases solved by the proposed EWCA are carried out in order to evaluate the efficiency of the proposed method in terms of power loss reduction and VSI improvement. Moreover, the proposed EWCA is compared with the existing approaches PSO, ELM, HSA, FWA, and ACSA indicated in the literature in order to show the effectiveness of the proposed method. For validation, the proposed EWCA has been examined on the IEEE 69- node radial distribution system. The simulation results obtained by the proposed cases reveal the significant power loss reduction from $55.73 \%$ to $84.2 \%$ with respect to the base case. In addition, the simulation results also show an acceptable improvement in the minimum voltage magnitude and voltage stability index $(3.7 \%-7.22 \%$ and $15.75 \%-29.7 \%$, respectively, compared with the base case).

The adopted algorithm can be easily modified to provide an integrated control algorithm and used to perform a network reconfiguration for larger distribution systems.

\section{References:}

[1] M. Karimi, H. Mokhlis, K. Naidu, S. Uddin, and A.H.A. Bakar, "Photovoltaic Penetration Issues and Impacts in Distribution Network A
Review", Renewable and Sustainable Energy Reviews, Vol.53, No.2, pp.594-605, 2016.

[2] O. Badran, S. Mekhilef, H. Mokhlis, and W. Dahalan, "Optimal Reconfiguration of Distribution System Connected with Distributed Generations: A Review of Different Methodologies", Renewable and Sustainable Energy Reviews, Vol.73, No.2, pp.854-867, 2017.

[3] R. AbulWafa, "A New Heuristic Approach for Optimal Reconfiguration in Distribution Systems", Electric Power Systems Research, Vol.81, No.2, pp.282-289, 2011.

[4] M. Abdelaziz, "Distribution Network Reconfiguration Using A Genetic Algorithm with Varying Population Size", Electric Power Systems Research, Vol.142, No.3, pp.9-11, 2017.

[5] I. I. Atteya, H. Ashour, N. Fahmi, and D. Strickland, "Radial Distribution Network Reconfiguration for Power Losses Reduction Using A Modified Particle Swarm Optimisation", CIRED - Open Access Proceedings Journal, Vol.2017, No.1, pp.25052508, 2017.

[6] D. Nataraj, R. Loganathan, M. Veerasamy, and V. Jawalkar, "Optimizing Radial Distribution System for Minimizing Loss Reduction and Voltage Deviation Indices Using Modified Grey Wolfs Algorithm", International Journal of Intelligent Engineering and Systems, Vol.11, No.6, pp.177-188, 2018.

[7] A. Mohamed Imran and M. Kowsalya, "A New Power System Reconfiguration Scheme for Power Loss Minimization and Voltage Profile Enhancement Using Fireworks Algorithm", International Journal of Electrical Power \& Energy Systems, Vol.62, No.6, pp.312- 322, 2014.

[8] S.-Ehsan Razavi, E. Rahimi, M. S. Javadi, A. E. Nezhad, M. Lotfi, M. Shafie-khah, and J. P.S. Catalo, "Impact of Distributed Generation on Protection and Voltage Regulation of Distribution Systems: A Review", Renewable and Sustainable Energy Reviews, Vol.105, No.20, pp.157-167, 2019.

[9] C. K. Das and O. Bass, "Optimal Placement of Distributed Energy Storage Systems in Distribution Networks Using Artificial Bee Colony Algorithm", Applied Energy, Vol.232, No.6, pp.212- 228, 2018.

[10] D. B. Prakash and C. Lakshminarayana, "Multiple DG Placements in Radial Distribution System for Multi Objectives Using Whale Optimization Algorithm", Alexandria 
Engineering Journal, Vol.57, No.8, pp.27972806, 2018.

[11] P. Mehta, P. Bhatt, and V. Pandya, "Optimal Selection of Distributed Generating Units and Its Placement for Voltage Stability Enhancement and Energy Loss Minimization", Ain Shams Engineering Journal, Vol.9, No.12, pp.187-201, 2018.

[12] M. S. Rawat and S. Vadhera, "Heuristic Optimization Techniques for Voltage Stability Enhancement of Radial Distribution Network with Simultaneous Consideration of Network Reconfiguration and DG Sizing and Allocations", Turk J Elec Eng. \& Comp. Sci., Vol.27, No.1, pp.330- 345, 2019.

[13] Z. Gong, Q. Chen, and K. Sun, "Novel Methodology Solving Distribution Network Reconfiguration with DG Placement", The Journal of Engineering, Vol. 2019, No. 16, pp. 1668-1674, 2019.

[14] R. S. Rao, K. Ravindra, K. Satish, and S. V. L. Narasimham, "Power Loss Minimization in Distribution System Using Network Reconfiguration in The Presence of Distributed Generation", IEEE Transactions on Power Systems, Vol.28, No.1, pp.317-325, 2013.

[15] A. M. Imran, M. Kowsalya, and D.P. Kothari, "A Novel Integration Technique for Optimal Network Reconfiguration and Distributed Generation Placement in Power Distribution Networks", International Journal of Electrical Power \& Energy Systems, Vol.63, No.12, pp.461-472, 2014.

[16] T. T. Nguyen, A. V. Truong, and T. A. Phung, "A Novel Method Based on Adaptive Cuckoo Search for Optimal Network Reconfiguration and Distributed Generation Allocation in Distribution Network", International Journal of Electrical Power \& Energy Systems, Vol.78, No.12, pp.801-815, 2016.

[17] I. B. Hamida, S. B. Salah, F. Msahli, and M. F. Mimouni, "Optimal Network Reconfiguration and Renewable DG Integration Considering Time Sequence Variation in Load and DGs", Renewable Energy, Vol.121, No.8, pp.66-80, 2018.

[18] B. J. Rani and A. S. Reddy, "Optimal Allocation and Sizing of Multiple DG in Radial Distribution System Using Binary Particle Swarm Optimization", International Journal of Intelligent Engineering \& Systems, Vol.12, No.1, pp.290-299, 2019.

[19] H. Eskandar, A. Sadollah, A. Bahreininejad, and M. Hamdi, "Water Cycle Algorithm A Novel Meta Heuristic Optimization Method for
Solving Constrained Engineering Optimization Problems", Computer Structure, Vol.110, No.2, pp.151-166, 2012.

[20] A. A. A. El-Ela, R. A. El-Sehiemy, and A. S. Abbas, "Optimal Placement and Sizing of Distributed Generation and Capacitor Banks in Distribution Systems Using Water Cycle Algorithm", IEEE Systems Journal, Vol.12, No.4, pp.3629- 3636, 2018.

[21] A. Sadollah, H. Eskandar, and J. H. Kim, "Water Cycle Algorithm for Solving Constrained MultiObjective Optimization Problems", Applied Soft Computing, Vol.27, No.4, pp.279-298, 2015.

[22] J. Wang and S. Liu, "Novel Binary Encoding Water Cycle Algorithm for Solving Bayesian Network Structures Learning Problem", Knowledge-Based Systems, Vol.150, No.4, pp.95-110, 2018.

[23] K. Yanjun, M. Yadong, L. Weinan, W. Xianxun, and B. Yue, "An Enhanced Water Cycle Algorithm for Optimization of Multi-Reservoir Systems", In: Proc. of 2017 IEEE/ACIS 16th International Conf. on Computer and Information Science (ICIS), pp.379-386, 2017.

[24] A. M. Imran and M. Kowsalya, "A New Power System Reconfiguration Scheme for Power Loss Minimization and Voltage Profile Enhancement Using Fireworks Algorithm", International Journal of Electrical Power \& Energy Systems, Vol.62, No.2, pp.312-322, 2014.

[25] M. Chakravorty and D. Das, "Voltage Stability Analysis of Radial Distribution Networks", International Journal of Electrical Power \& Energy Systems, Vol.23, No.2, pp.129-135, 2001.

[26] S. Jena and S. Chauhan, "Solving Distribution Feeder Reconfiguration and Concurrent DG Installation Problems for Power Loss Minimization by Multi Swarm Cooperative PSO Algorithm", In: Proc. of 2016 IEEE/PES Transmission and Distribution Conf. and Exposition ( $T \& D)$, Dallas, TX, USA, pp. 1-9, 2016.

[27] The American National Standards Institute (ANSI), "American National Standard for Electric Power Systems and Equipment Voltage Ratings (60) Hertz, NSI, C84.1-1995, 1996.

[28] N. C. Sahoo and K. Prasad, "A Fuzzy Genetic Approach for Network Reconfiguration to Enhance Voltage Stability in Radial Distribution Systems", Energy Conversion and Management, Vol.47, No.18, pp.3288-3306, 2006.

[29] W. H. Kersting, Distribution System Modeling and Analysis, Vol.3, CRC Press, Boca Raton, N.Y. 2015. 